\title{
Distribution d'anneaux vaginaux contraceptifs
}

Population Council

Follow this and additional works at: https://knowledgecommons.popcouncil.org/departments_sbsr-rh

Part of the Demography, Population, and Ecology Commons, Family, Life Course, and Society Commons, International Public Health Commons, and the Women's Health Commons How does access to this work benefit you? Let us know!

\section{Recommended Citation}

"Distribution d'anneaux vaginaux contraceptifs," fact sheet. New York: Population Council, 2015. 


\section{DISTRIBUTION D'ANNEAUX VAGINAUX CONTRACEPTIFS}

Les anneaux vaginaux contraceptifs aident l'USAID à résoudre un problème important: le besoin non satisfait de planification familiale

Pour soutenir une distribution et une utilisation durables des contraceptifs, I'USAID a récemment proposé un nouvel objectif, articulé autour de cinq éléments clés pour répondre aux besoins non satisfaits de planification familiale : 1) Choix de la méthode, 2) Approche de Marché total, 3) Personnel de planification familiale, 4) Communication pour le changement de comportement et 5) Systèmes d'approvisionnement renforcés.

Dans le cadre de son projet "Distribution d'anneaux vaginaux contraceptifs", le Population Council a conclu un accord de coopération avec I'USAID pour introduire deux nouvelles méthodes contraceptives modernes sur les marchés en développement : l'anneau vaginal contraceptif à progestérone (AVP), un anneau contraceptif d'une durée d'action de trois mois spécifiquement conçu pour espacer les grossesses durant l'allaitement et un anneau vaginal contraceptif expérimental d'une durée d'utilisation d'un an - l'anneau vaginal contraceptif Nestorone ${ }^{\circledR} /$ Ethinyl Estradiol (NES/EE CVR) - qui peut être utilisé par les femmes sexuellement actives en âge de reproduction (15 à 49 ans) pour retarder ou espacer les grossesses.

Le Population Council a stratégiquement adopté les cinq éléments clés de I'USAID pour introduire l'AVP et le NES/EE CVR sur les marchés en développement.

\section{CHOIX DE LA MÉTHODE}

L'AVP et le NES/EE CVR joueront un rôle essentiel pour élargir le choix et accroître l'accès à la contraception pour des millions de femmes. Les caractéristiques uniques des anneaux vaginaux contraceptifs répondent aux besoins des utilisatrices et pallient le manque de produits contraceptifs. Les anneaux sont de petite taille, flexibles et peuvent être facilement insérés et retirés pour éviter une grossesse.

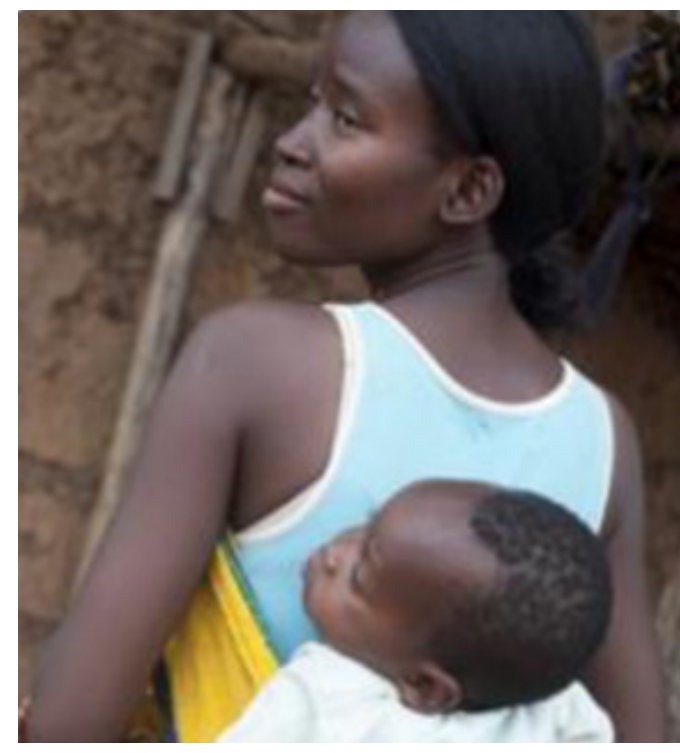

Le Population Council est le premier concepteur d'une contraception durable et réversible.

Les produits développés par le Population Council incluent le stérilet Cooper T, le dispositif intrautérin libérant du lévonorgestrel Mirena $\AA$, le Jadelle et Norplant $\AA$. De nouveaux produits en développement incluent les anneaux vaginaux contraceptifs, les gels et les produits de contraception pour les hommes.

L'AVP et le NES/EE CVR sont des méthodes contraceptives non cliniques qui peuvent être contrôlées par les femmes elles-mêmes et, par conséquent, exigent une intervention minimale de l'utilisatrice ainsi que du prestataire (contrairement aux méthodes cliniques telles que les produits injectables, stérilet et implants). De plus, les anneaux sont discrets et ne gênent pas l'acte sexuel. Les résultats des essais cliniques récents indiquent que la plupart des couples ne sentent pas l'anneau durant un rapport sexuel et ceux qui l'ont senti, n'ont pas été gênés par sa présence.

Le Population Council mène des recherches et offre des solutions qui améliorent des vies dans le monde entier. De grandes idées qui apportent leur preuve : c'est notre philosophie pour un changement global. www.popcouncil.org

(C) 2015 The Population Council, Inc.

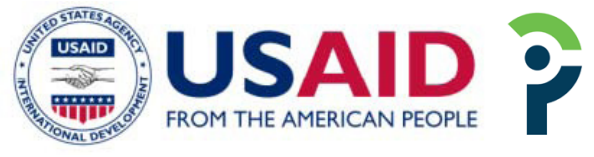

POPULATION COUNCIL

Ideas. Evidence. Impact. 
Le Population Council vise à élargir le choix des méthodes en introduisant les contraceptifs dans des pays sélectionnés à faible et moyen revenu, dans un contexte de droit et de choix.

\section{PRESTATAIRES DES SERVICES DE PLANIFICATION FAMILIALE}

Le problème crucial de la prestation de services de planification familiale est le manque de professionnels de la santé, surtout en Afrique sub-saharienne, où il y a moins de 2,3 professionnels de santé pour 1000 personnes (données de l'OMS 2006 et 2010). Cette pénurie de travailleurs expérimentés limite l'accès aux services et aux produits de contraception.

La fourniture non clinique de l'AVP permet sa distribution par des catégories inférieures de prestataires de soins de santé à la fois dans les communautés et les établissements de soins. Suivant les directives de l'OMS "Optimisation des rôles du personnel de santé par la délégation des tâches pour améliorer l'accès aux interventions de santé maternelle et néonatale", le Council incorporera des mécanismes de partage et de délégation des tâches pour distribuer les anneaux en ayant recours à des prestataires de faible et moyen niveau.

L'approche de la délégation des tâches aide les prestataires compétents de niveau supérieur à maximiser leur temps pour les méthodes exigeant un approvisionnement clinique. De plus, I'AVP et le NES/EE CVR peuvent être distribués par des agents de santé communautaires, ce qui permettra de réduire le coût de fourniture du service et d'élargir l'accès. Les clients auront besoin de conseils basiques sur l'utilisation de l'anneau vaginal.

\section{APPROCHE DE MARCHÉ TOTAL}

Pour atteindre les populations ciblées dans les pays en développement, le Population Council doit oeuvrer dans tout l'environnement du marché contraceptif, incluant les secteurs public, privé-commercial et privé à but non lucratif.

Les anneaux contraceptifs peuvent être distribués à travers de multiples canaux, dont les pharmacies, les agents de santé communautaires, les dépositaires, les franchises sociales et les établissements de soins de santé traditionnels.

La stratégie de distribution à travers le marketing social des AVP, exploitera un vaste champ d'initiatives et de partenariats publics et privés pour atteindre toute la clientèle à travers l'approche de marché total, depuis ceux qui nécessitent des services gratuits ou des tarifs subventionnés à ceux qui peuvent et veulent payer pour les produits. Les anneaux contraceptifs peuvent être inclus dans des paquets de services qui sont couverts par des systèmes de bons, des canaux de marketing social et en regroupant et intégrant les services.

\section{COMMUNICATION POUR LE CHANGEMENT DE COMPORTEMENT}

Des efforts pour promouvoir un changement de comportement

Du fait que l'AVP est une nouvelle méthode de contraception, plusieurs outils de communication et matériels informationnels seront développés dans une variété de formats pour éduquer les consommateurs, les communautés, les prestataires de services et les acteurs principaux.
(CCC) seront adoptés non seulement pour autonomiser les femmes et les informer sur leurs options contraceptives (incluant des informations sur les avantages des anneaux vaginaux contraceptifs) mais aussi pour autonomiser et créer un environnement approprié pour un accès et une utilisation durables.

Un manuel de marketing et un plan de communication en direction des parties prenantes sont en cours de développement, et décrivent les stratégies de promotion et de mise en oeuvre parmi les acteurs et les bénéficiaires. Par exemple, les matériels de communication de promotion des anneaux contraceptifs (incluant les affiches, les dépliants et les banderoles) seront conçus pour être distribués par les prestataires de soins de santé et les agents de santé communautaires. Des policy brief et une déclaration sur la valeur ajoutée apportée des anneaux contraceptifs sont en cours de formulation pour engager les acteurs clés des ministères de la santé et les organisations de plaidoyer. Des matériels de formation, des programmes de counseling et des outils seront intégrés dans les programmes de planification familiale des pays où les anneaux contraceptifs sont introduits.

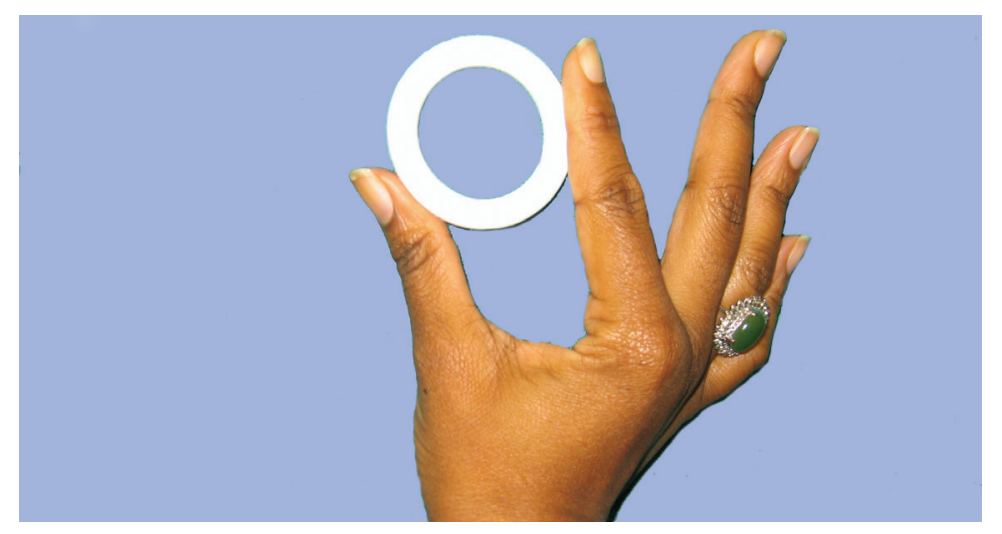

\section{SYSTÈMES D'APPROVISIONNEMENT}

Reconnaissant que de nouvelles technologies doivent être intégrées dans des systèmes existants d'approvisionnement et de logistique, le Council s'engage à identifier des canaux principaux de distribution et d'approvisionnement locaux et à faire en sorte qu'ils communiquent efficacement avec les fabricants agréés pour les deux anneaux contraceptifs.

Le Council collaborera avec des organismes d'achat globaux établis tels que I'UNFPA, Crown Agents, DKT, et des agences gouvernementales centrales pour garantir des volumes d'achat élevés, des coûts faibles, des services d'expédition améliorés et une gestion optimale des stocks. Un système local de gestion logistique (USAID/DELIVER PROJECT 2011) sera identifié ou développé pour assurer un approvisionnement constant de l'AVP et NES/EE CVR aux divers points de prestations de service.

\section{Références}

USAID. 2011. "The Logistics Handbook: A Practical Guide for the Supply Chain Management of Health Commodities." Arlington, VA: USAIDI DELIVER PROJECT.

WHO. 2010. "Achieving the health-related MDGs. It takes a workforce!" www.who.int/hrh/workforce_mdgs/en/.

_. 2006. "New global alliance seeks to address worldwide shortage of doctors, nurses and other health workers." www.who.int/ mediacentre/ news/releases/2006/pr26/en/ 